\title{
Trayectorias de mujeres bolivianas en áreas rurales y urbanas de Mendoza (Argentina)
}

\author{
Bolivian women pathways in rural and urban areas \\ of Mendoza (Argentina)
}

\author{
Marta Silvia Moreno* \\ Áridas (IADIZA), CONICET / Facultad de Ciencias Políticas y Sociales, \\ Universidad Nacional de Cuyo / Universidad Nacional de Chilecito
}

\begin{abstract}
María Victoria Martínez Espínola**
Instituto de Ciencias Humanas, Sociales y Ambientales (NCISHUSA), CONICET / Facultad de Ciencias Políticas y Sociales, Universidad Nacional de Cuyo
\end{abstract}

Recibido: 9 de junio de 2016. Aprobado: 06 de Febrero de 2017

\begin{abstract}
Resumen
El presente trabajo intenta explorar las potencialidades de un abordaje cuali-comparativo de las trayectorias diferenciales de mujeres bolivianas que residen actualmente en la provincia de Mendoza y se desempeñan en dos segmentos del mercado de trabajo destinados a migrantes recientes, como lo son la agricultura en contextos rurales y el comercio informal en espacios urbanos. Desde una perspectiva de género, analizamos las trayectorias migratorias y laborales de estas mujeres a partir de dos investigaciones realizadas entre 2010 y la actualidad en Ugarteche (distrito de Luján de Cuyo que convoca a trabajadores/as agrícolas) y Belgrano (distrito urbano de Guaymallén, departamento del Gran Mendoza, donde tiene un fuerte peso el comercio en ferias emplazadas en espacios públicos). Así, buscamos poner en diálogo algunos hallazgos de investigación basados en distintos contextos laborales y espaciales, que comparten algunas dimensiones relevantes,

* Doctoranda en Ciencias Sociales en Facultad de Ciencias Políticas y Sociales, Universidad Nacional de Cuyo / Universidad Nacional de Chilecito / Instituto Argentino de Investigaciones de Zonas Áridas (IADIZA), CONICET. Correo electrónico: smoreno@mendoza-conicet.gob.ar

* Doctoranda en Ciencias Sociales en Facultad de Ciencias Políticas y Sociales, Universidad Nacional de Cuyo / Instituto de Ciencias Humanas, Sociales y Ambientales (NCISHUSA), CONICET. Correo electrónico: viquimartinez@hotmail.com
\end{abstract}


como la unidad temporal de investigación, la metodología empleada, los lugares de procedencia de las entrevistadas y sus grupos de edad.

Palabras clave: migración, trayectorias, género.

\begin{abstract}
This paper attempts to explore the potential of a qualitative and comparative approach regarding the differential trayectories of Bolivian women who live in the province of Mendoza and work in two segments of the labor market aimed at recent immigrants, such as agriculture in rural contexts and informal trade in urban spaces. From a gender perspective, we analyzed the migratory and labor trajectories of these women from two researches, conducted between 2010 and today in Ugarteche (district of Luján de Cuyo that demands agricultural workers) and Belgrano (urban district of Guaymallén department of Mendoza, where trade it has a strong weight emplaced in public spaces). Thus, we seek to articulate some research findings based on different labor contexts and spatial units, which share some relevant dimensions, such as temporary research unit, the methodology used, the places of origin of respondents and their age groups.
\end{abstract}

Keywords: migration, trajectories, gender.

\title{
Introducción
}

Las migraciones desde Bolivia hacia Argentina constituyen un flujo poblacional de larga data, que en Mendoza se remonta a mediados del siglo pasado, revistiendo actualmente un considerable dinamismo. Gran parte de los desplazamientos que tuvieron lugar desde comienzos del siglo XX constituyeron migraciones laborales (Pizarro et al., 2011) con destino hacia ciertos segmentos del mercado de trabajo destinados a "migrantes recientes" (Herrera Lima, 2005). La teoría de la segmentación del mercado de trabajo es un marco analítico de referencia para analizar la inserción laboral de las/os extranjeras/os (Piore, 1979) que parte de considerar que la dinámica del capitalismo moderno conduce a que los puestos de trabajos más inestables y peor remunerados tiendan a ser ocupados por trabajadoras y trabajadores migrantes (Herrera Lima, 2005). En el contexto nacional, 
se ha comprobado que históricamente estas/os migrantes han tenido una inserción marginal en el mercado de trabajo argentino, que resultaba funcional a la demanda de empleos de baja calificación, especialmente en el sector informal (Benencia, 2008). Esta tendencia persiste en las primeras décadas del siglo XXI, consolidando un patrón de inserción laboral segmentada que permitió su refugio en determinados sectores, como la agricultura, la construcción, el comercio ambulante, las pequeñas industrias y los cortaderos de ladrillos (Sassone, 2009; Pizarro et al., 2011).

En el caso de la provincia de Mendoza, estos nichos laborales se configuraron inicialmente alrededor de la demanda estacional de trabajo en las cosechas agrícolas, una actividad desvalorizada en el contexto local por su exigencia física y bajos salarios en el marco de acuerdos predominantemente informales (Moreno, 2012, 2013; Moreno y Torres, 2013; Pizarro y Moreno, 2015). Si primeramente se trató de una movilidad estacional conformada mayoritariamente por varones solos, con el tiempo se fue incrementando la participación de las mujeres en el marco de migraciones familiares (incluyendo entre estas a las migraciones de mujeres con hijos/as a cargo y sin cónyuges), tendiendo algunas familias a radicarse en diversas localizaciones rurales y urbanas de la provincia. Junto al paulatino establecimiento de estas/os migrantes en Mendoza, la venta callejera al menudeo o en ferias emplazadas en espacios públicos se posicionaron como un destino relevante para las mujeres (García Vázquez, 2005; Martínez Espínola, 2010, 2016; Colque et al., 2015).

En este marco, buscamos poner de relieve el protagonismo de las mujeres y la especificidad de sus experiencias en estos procesos de movilidad territorial con la finalidad de superar el reduccionismo economicista que asocia la migración laboral únicamente al trabajo productivo de los varones (Trpin y Radonich, 2013). Coincidimos en cuestionar aquellas perspectivas homocentristas en los estudios migratorios, que fijan su atención en los varones y conciben a las mujeres como acompañantes o seres dependientes incapaces de llevar adelante un proyecto migratorio, con el objetivo de superar el sesgo que Beatriz Padilla denomina "sexismo metodológico", el cual ha permeado hasta no hace mucho tiempo a la investigación en ciencias sociales en general y sobre migraciones en particular (Padilla, 2013, p. 2). La estrategia metodológica elegida es el análisis cuali-comparativo de trayectorias laborales de mujeres bolivianas que residen actualmente en la provincia de Mendoza. Nos proponemos comprender estas trayectorias en dos segmentos del mercado de trabajo, como lo son la agricultura en contextos rurales y la venta en espacios urbanos. Realizamos este abordaje a partir de dos investigaciones realizadas entre 2010 y la actualidad en Mendoza. Una de ellas fue efectuada en el distrito de Ugarteche (Luján de Cuyo), donde residen/ transitan y trabajan estacionalmente un considerable número de trabajadoras/es agrícolas. La otra, realizada en el distrito Belgrano (Guaymallén), ubicado en el Gran Mendoza, 
donde el comercio en ferias emplazadas en espacios públicos alcanza una importante difusión. Así, buscamos poner en diálogo algunos hallazgos de dos investigaciones basadas en distintas unidades espaciales, que comparten algunas dimensiones de análisis tales como la unidad temporal de la investigación, la aproximación teórico-metodológica, los lugares de procedencia de las entrevistadas y sus grupos de edad. La anticipación de sentido que guía la pesquisa señala múltiples puntos de entrecruzamiento en las trayectorias laborales de las mujeres entrevistadas, así como ciertas especificidades en las elecciones laborales de acuerdo a los contextos rurales o urbanos donde residen.

\section{Breves notas sobre la migración boliviana en Mendoza}

Los desplazamientos de personas nacidas en los países vecinos hacia Argentina tienen una larga historia (Balan, 1985; Benencia, 2004). En el caso particular de la migración boliviana, la misma antecede la conformación de los estados republicanos, pero solo reviste un carácter laboral desde comienzos del siglo XX (Hinojosa Gordonava, 2009), cuando los campesinos del sur y los valles de Bolivia comenzaron a trasladarse estacionalmente a las zonas fronterizas del noroeste de Argentina para trabajar en la zafra cañera y en las cosechas de tabaco (Balán, 1985; Whiteford, 2001).

A partir de 1950 estos desplazamientos se ampliaron hacia áreas más distantes en el territorio nacional, entre ellas la provincia de Mendoza, destacada por sus requerimientos en las labores estacionales de las cosechas agrícolas, principalmente de vid (García Vázquez, 2005). Estas migraciones se vieron favorecidas por el fracaso de la reforma agraria boliviana en 1952 y la consecuente emigración de muchos campesinos indígenas hacia el exterior de Bolivia (Pizarro, 2013). También incidió la construcción de un nuevo ramal ferroviario que vinculó a Mendoza con el norte de Argentina y con Bolivia, proporcionando una vía de transporte rápida para el traslado (Paredes, 2004). Progresivamente, en los alrededores de la estación San José, ubicada en la ciudad de Mendoza, se instalaron nuevos inmigrantes, en su mayoría procedentes de Bolivia (Paredes, 2004; García Vázquez, 2005). Algunos buscaron nuevas posibilidades laborales en la capital y sus alrededores (García Vázquez, 2005), mientras que otros se dirigieron a las zonas rurales, motivados por el incremento constante de la superficie cultivada y por los lugares vacantes que dejaba la población nativa en su camino hacia las metrópolis (Cavagnaro y Balussi, 1962) en busca de mejores condiciones de vida y de nuevas oportunidades de trabajo. 
$\mathrm{Si}$ en un primer momento se trataba de una movilidad estacional conformada mayoritariamente por varones solos, con el tiempo se fue incrementando la participación de las mujeres en el marco de migraciones familiares. Entre las décadas de 1970 y 1980 creció la tendencia hacia la fijación residencial en zonas urbanas y rurales en diferentes puntos del país, con particular énfasis en el Área Metropolitana de Buenos Aires. Se diversificaron los destinos y se ampliaron los desplazamientos dentro de Argentina, constituyéndose Mendoza en el tercer destino en importancia.

A partir de la década de 1990 la migración boliviana hacia Argentina se incrementó, alcanzando el segundo lugar en importancia a nivel nacional, luego de la paraguaya. En Mendoza, este crecimiento ha posicionado a las/os migrantes bolivianos como la primer minoría de extranjeras/os en la provincia desde 2010 (DEIE, CNPHyV 2010). Esta misma fuente indica, además, un leve aumento en la proporción de mujeres bolivianas respecto al Censo de 2001 (representando el 48,9\% de la población boliviana en Mendoza), aunque los varones mantienen una participación levemente mayor.

El acercamiento sobre los fenómenos migratorios en Argentina y Mendoza sirve de punto de partida para avanzar en la especificación de las categorías teóricas clave que estructuran el cuerpo de este trabajo. Dichos conceptos se utilizarán para la construcción y el análisis cuali-comparativo de las trayectorias laborales de las trabajadoras bolivianas entrevistadas.

\section{Algunas precisiones conceptuales para el análisis de las trayectorias migratorias y laborales desde una perspectiva de género}

Para el análisis de las trayectorias laborales partimos de una concepción ampliada de trabajo $^{1}$ (Garza Toledo, 2002; Herrera Lima, 2005), dado que su especificidad no proviene de las características del objeto ni de las actividades mismas, sino de la articulación de este proceso de producción con determinadas relaciones sociales amplias que involucran relaciones económicas, de poder, de influencia y culturales (Garza Toledo, 2002). Asimismo, el trabajo en la agricultura, como la venta ambulante al menudeo o en ferias, es abordado desde la teoría de la segmentación del mercado de trabajo (Piore, 1979) o de conformación

1 En este sentido, las indagaciones sobre economías informales o sumergidas, así como los aportes que provienen de las teorías feministas (Narotzky, 2004), han representado una importante contribución en la redefinición de este concepto. Estos abordajes puntualizan su crítica respecto de una concepción restringida de trabajo fuertemente vinculada a la noción de empleo, que entre otras cosas ha contribuido a invisibilizar y desvalorizar las inserciones informales y el trabajo productivo/reproductivo de las mujeres (Comas D’Argemir, 1998; Gregorio Gil, 2011). 
de "nichos laborales para migrantes recientes", que se caracterizan por "reproducir de manera cíclica algunas franjas del mercado laboral [...] por el constante arribo de nueva fuerza de trabajo migrante" (Herrera Lima, 2005, p.168). Entre ambas actividades, el trabajo estacional agrícola constituye un destino "tradicional" para las/os migrantes procedentes de Bolivia y el noroeste de Argentina. Este se desarrolla en el contexto rural de la provincia, donde tienen preeminencia las actividades que componen el sector primario de la economía, que comprende las actividades productivas de extracción y obtención de materias primas, entre ellas la agricultura. Se caracteriza por constituir una modalidad casi enteramente informal de trabajo asalariado que se remunera por productividad (a destajo) y al que se accede a través de diversas formas de intermediación, donde destaca la figura de los "cuadrilleros" (Moreno, 2012, 2015). Por su parte, la venta ambulante o en ferias tiene lugar en el contexto urbano de la provincia, donde se concentran las actividades y el empleo en los sectores secundario (industrial) y terciario (servicios). Se trata de una modalidad de autoempleo no asalariado en la que los ingresos obtenidos surgen de la diferencia entre el precio de compra y venta de una amplia diversidad de productos.

Dentro del campo de los estudios migratorios existe abundante conocimiento acumulado sobre las vinculaciones entre género y trabajo. Por ejemplo, la segregación por género en lo que respecta a los puestos de trabajo y los niveles de ingreso resulta una característica arraigada y dominante (Hondagneu-Sotelo, Estrada y Ramírez, 2011)2. En este sentido, nos resulta esclarecedora la noción de género propuesta por Joan Scott (1990), para quien el concepto de género consta de la integración de dos proposiciones fundamentales: el género es un elemento constitutivo de las relaciones sociales basadas en las diferencias que distinguen los sexos y es una forma primaria de relaciones significantes de poder. Es decir, el género constituye el campo primario dentro del cual o por medio del cual se articula el poder. Para analizar los procesos de construcción de las relaciones de género, Scott señala cuatro aspectos que se interrelacionan: símbolos culturales que evocan representaciones múltiples y contradictorias (relatos tradicionales); conceptos normativos (interpretaciones de los símbolos) que afirman unívocamente el significado de lo masculino y lo femenino (religiones, teorías científicas, leyes); nociones políticas que circulan en instituciones y organizaciones sociales (política, educación, mercado de trabajo); identidades subjetivas (psicoanálisis, biografías) (Scott, 1990, pp. 44-46). Si bien entendemos que todas estas dimensiones se presentan entrelazadas y que solo pueden distinguirse a los fines analíticos, en el marco puntual de este trabajo hemos privilegiado dos de los aspectos que menciona Scott para analizar la dimensión de

2 De acuerdo con Gregorio Gil (2003), las investigaciones sobre migración y género constituyen una continuidad de la perspectiva de la antropología feminista de la década de 1970, que hacía foco sobre las relaciones entre el capitalismo internacional y su impacto diferencial según el género. 
género: fundamentalmente el mercado de trabajo, entendido como una dimensión de la organización social que vivimos atravesada por desigualdades de género y la dimensión subjetiva, basándonos en las narrativas de las entrevistadas. Por otro lado, desde las aproximaciones que analizan la vinculación entre género y migración, se pone énfasis en examinar el trabajo tanto en su esfera productiva y remunerada, como también en aquella reproductiva y no remunerada (Comas D’Argemir, 1998; Gregorio Gil, 2011). Una importante contribución en este sentido han sido las investigaciones sobre el trabajo doméstico como paradigma "natural" del trabajo reproductivo de las mujeres migrantes, aspectos analizados sobre la base de novedosos conceptos ("trabajo de cuidado", cadenas globales de cuidados y afectos, maternidad transnacional y trabajo de reproducción social) (Hondagneu-Sotelo, Estrada y Ramírez, 2011). En el presente trabajo buscamos, sin embargo, analizar las trayectorias de las mujeres migrantes desde su articulación al trabajo "productivo", sin por ello desconocer las obligaciones reproductivas que pesan sobre sus hombros. En este sentido, los resultados de algunas investigaciones señalan que gran parte de las mujeres bolivianas que se articulan al trabajo productivo en Argentina desarrollan las actividades extra-domésticas en conjunto con las tareas domésticas (Magliano y Mallimaci, 2015). Es decir, el trabajo en la agricultura, como la venta al menudeo, entra en diálogo con las tareas reproductivas que involucran desde limpiar, lavar la ropa y cocinar hasta cubrir el cuidado de pequeños y ancianos.

Vemos así que tomar en consideración a la familia no solo reviste importancia en el análisis de la esfera reproductiva, sino que además resulta central en el abordaje de los proyectos migratorios. En este sentido, Parella (2007) sostiene que aun cuando en muchos casos las/os migrantes se desplazan solas/os por el territorio, estas decisiones no suelen tomarse en forma aislada o individual (Parella, 2007). Por el contrario, forman parte de proyectos migratorios familiares, o bien están enmarcadas en relaciones de parentesco que pueden estar atravesadas tanto por relaciones asimétricas de poder como de reciprocidad. Lo que queremos destacar, siguiendo a Medeiros de Melo y Moraes Silva (2012), es que un análisis de la trayectoria a partir de un individuo debe siempre tener en cuenta la trayectoria familiar, puesto que la primera es, inevitablemente, también producto de la dinámica de las relaciones de fuerza que frecuentemente recomponen el grupo familiar y reorganizan las acciones y experiencias individuales. Desde esta perspectiva, las familias no son entendidas como unidades biológicas per se, sino como construcciones sociales o "comunidades imaginadas" que deben hacer frente a las relaciones de poder y de desigualdad en el acceso a los recursos que se dan en su seno (Bryceson y Vuorela, 2002, pp. 3-7; en Parella, 2007, p. 155). En tal caso, se trata de familias migrantes que no necesariamente responden al modelo de familia moderna corresidencial, nuclear 
y conyugal (Trpin y Radonich, 2013; Malimacci Barral, 2013), englobando una mayor heterogeneidad de situaciones ${ }^{3}$.

Este marco analítico permite poner de relieve las estructuras patriarcales sedimentadas históricamente que legitiman los derechos y autoridad naturales del patriarca dentro de la familia (Harris, 1986). Ariza y Oliveira (2001) destacan que uno de los principales aportes de las teorías de género al estudio de la familia es su llamado de atención sobre el carácter asimétrico de las relaciones intergenéricas e intergeneracionales, que cuestionan la visión ideologizada de la familia como entidad armónica y cohesionada. Las autoras sostienen que a partir de estos cuestionamientos surge el interés por analizar la dinámica intrafamiliar como el conjunto de relaciones de cooperación, intercambio, poder y conflicto que hombres y mujeres de diferentes generaciones establecen en el seno de las familias en torno a los procesos de organización de la vida familiar (división del trabajo, toma de decisiones) y de los procesos migratorios.

Además, para el caso particular de las mujeres provenientes de Bolivia, resultan muy esclarecedoras las reflexiones de Rivera Cusicanqui (2010) sobre los sistemas de género en las sociedades andinas, que conceptualiza como fruto de la articulación y simultaneidad entre distintos horizontes históricos, desde el pasado prehispánico hasta la época actual. A través de la superposición de temporalidades podemos comprender por qué perduran hasta la actualidad las consecuencias de la colonización en diversos espacios de la vida social, incluidas las relaciones de género y cómo estas últimas se encuentran unidas "umbilicalmente" al patriarcado en las sociedades andinas (Rivera Cusicanqui, 2010, p. 190).

A continuación realizaremos algunas precisiones metodológicas sobre nuestro abordaje, para luego avanzar en el análisis cuali-comparativo de las trayectorias migratorias y laborales de mujeres procedentes de Bolivia.

3 Esta perspectiva contradice la idílica visión parsoniana de la familia nuclear basada en el jefe varón proveedor exclusivo y la mujer-ama de casa, para visibilizar la creciente presencia de hogares con dos proveedores y de familias monoparentales o encabezadas por mujeres (Ariza y Oliveira, 2001). 


\section{Algunas reflexiones metodológicas para la construcción de los datos de campo}

Lainformación aportada sobrelas trayectorias se construyó a partir delenfoque etnográfico (Hammersley y Atkinson, 1994), y dentro del conjunto de técnicas disponibles en este enfoque, nos servimos de los "relatos de vida" de nuestras informantes4. Así, por medio de entrevistas etnográficas buscamos capturar y comprender las prácticas y sentidos que se construyeron alrededor de los desplazamientos y de las inserciones laborales, a partir de fuentes primarias de información, en tanto instrumentos claves para restituir la perspectiva o voz de nuestras interlocutoras.

En esta dirección, se abordó el encadenamiento entre las sucesivas posiciones que las entrevistadas ocuparon a lo largo de su itinerario, tomando en consideración las trayectorias familiares (Moraes Silva y Menezes, 2011) y laborales (Herrera Lima, 2005), así como las motivaciones, expectativas y representaciones que rodearon los procesos de movilidad (Pedreño Cánovas, 2006).

En dicho acercamiento tuvimos en cuenta que la memoria es un conjunto de los marcos o esquemas sociales que condicionan los recuerdos y olvidos de un grupo social (Pizarro, 2006). Las memorias individuales cambian y se modifican dentro de estos universos de sentido, por lo que su puesta al día como "relatos de vida" es siempre provisoria (Candau, 2002). Para Moraes Silva y Menezes (2011), la vida actual de las personas posee un carácter activo en el desencadenamiento del curso de sus memorias. Consideran que el pasado no resurge por un proceso espontáneo, sino que supone un trabajo de rememoración que está siempre vinculado a acontecimientos y eventos presentes. De modo que entendemos que nuestras interlocutoras narraron determinados sucesos que fueron seleccionados y evaluados desde el presente de la enunciación, ya que el recuerdo siempre supone una relectura "que nos posibilita interpretar el rol que el pasado asume en el momento presente, y en lo que se puede vislumbrar de futuro" (Medeiros de Melo y Moraes Silva, 2012, p. 65).

Los datos del trabajo de campo provienen de dos investigaciones localizadas en la provincia de Mendoza que comenzaron en 2010 y todavía permanecen en curso. Una

4 Consideramos que esta estrategia metodológica resulta apropiada, dado que a diferencia de las historias de vida (o relato autobiográfico), "son narraciones biográficas acotadas al objetivo de estudio del investigador [que] si bien puede abarcar la amplitud de toda la experiencia de vida de una persona, empezando por su nacimiento, se centra en un aspecto particular de esta experiencia" (Kornblit, 2007, p. 16), que en nuestro caso se focalizó en la experiencia migratoria, laboral y territorial de las/os migrantes. 
de ellas tuvo lugar en el distrito de Ugarteche (Luján de Cuyo), donde residen/transitan y trabajan estacionalmente un considerable número de trabajadoras/es agrícolas. En la localidad se llevaron a cabo sucesivas instancias de observación participante en los espacios de socialización, así como en los de contratación y trabajo agrícola, con mayor énfasis durante el período 2012-2014. En estos espacios se efectuaron 18 relatos de vida sobre las trayectorias de siete mujeres y once varones provenientes de Bolivia, que nacieron en los campos de Potosí (12 casos), en áreas rurales de Cochabamba (3 casos), en el campo de Tarija (un caso), en el campo de Santa Cruz de la Sierra (un caso) y en la ciudad de Oruro (un caso), respectivamente. Se optó por efectuar conversaciones abiertas no estrictamente pautadas por un cuestionario rígido, con la finalidad de brindar libertad a las/os entrevistadas/os de rememorar y expresar sus opiniones, adaptando la técnica a las particularidades de cada caso.

La otra investigación fue realizada en el distrito Belgrano (Guaymallén), ubicado en el Gran Mendoza, donde la venta al menudeo en ferias emplazadas en espacios públicos alcanza una importante difusión. En dicho emplazamiento se efectuaron siete entrevistas a vendedoras del centro de la ciudad y cinco en el distrito de Belgrano a mujeres procedentes de Bolivia. El primer grupo estuvo conformado por mujeres nacidas en zonas rurales de Potosí (cuatro casos), en zonas rurales y urbanas de La Paz (dos casos) y en la ciudad de Cochabamba (un caso), mientras el segundo estuvo constituido por mujeres nacidas en Sucre (tres casos) y en Potosí (dos casos). Las entrevistas etnográficas fueron complementadas por sucesivas observaciones participantes en los espacios de trabajo en la feria, así como los barrios donde residen las entrevistadas a partir de la participación de numerosas festividades, celebraciones religiosas y otras instancias de encuentro, tales como talleres de alfabetización y talleres de educación popular sobre temas de género.

En este marco, intentamos poner en juego las configuraciones de las trayectorias laborales de mujeres que actualmente se desempeñan en contextos rurales y urbanos de Mendoza. Algunas de las preguntas nodales que orientaron estas pesquisas fueron las siguientes: ¿De qué lugar de Bolivia es usted? ¿Cómo fue el momento de salir de Bolivia? ¿Por qué decidió irse? ¿Cuál fue su itinerario hasta llegar a Mendoza y en qué lugares permaneció para vivir y trabajar? ¿Cómo fue su llegada a Mendoza? ¿Por qué decidió quedarse aquí? ¿A qué se dedicaba usted allá, y a qué se ha dedicado en Argentina? ¿Cómo se ha sentido usted, como mujer, en esta experiencia de viajar, cambiar de lugar de residencia? ¿A qué se dedicaban las mujeres de su familia en Bolivia (madre, hermanas, tías), ¿y aquí? ¿Cómo han sido sus experiencias laborales en Mendoza? 
Mediante la exposición y análisis cuali-comparativo de algunas experiencias migratorias diferenciales, a continuación intentaremos mostrar que muchas mujeres trabajadoras han sido más que simples "acompañantes" de sus maridos (Trpin y Radonich, 2013), para situarse en el lugar de gestoras de sus proyectos y trayectorias.

Consideramos que el intento por poner en diálogo nuestras investigaciones a partir de un análisis comparativo es una vía potente para avanzar hacia una comprensión holística del entrelazamiento entre trayectorias migratorias y laborales de mujeres bolivianas en Mendoza. Varios estudios sobre la temática migratoria demuestran esta potencialidad y nos animan a sumar esfuerzos en este sentido. Tal es el caso del análisis de Magliano, Perissinotti y Zenklusen (2013), quienes comparan las especificidades en los modos de inserción laboral en el servicio doméstico remunerado de mujeres bolivianas y peruanas en la ciudad de Córdoba, Argentina, a fin de comprender cómo el origen nacional, el género y la clase social condicionan estas trayectorias (Magliano et al., 2013, p. 72). Otro análisis que arroja resultados comparativos es el de Núñez Borja y Stallaert (2013) sobre trayectorias migratorias diferenciales de mujeres andinas en Bruselas, Bélgica, atendiendo al género, la etnicidad y la clase social entendida como ubicación social "en función al acceso a educación, ocupación, estilo de vida e ingreso económico" (Núñez Borja y Stallaert, 2013, p. 36). Por último, el estudio de Carolina Rosas (2013) acerca de trayectorias laborales y migratorias de varones y mujeres latinoamericanos/as en diversos espacios geográficos nos permite dimensionar cómo el ejercicio comparativo puede ampliarse en términos de género al colectivo de varones, enfoque que muestra aún muchas ausencias en el campo. Así, frente a la diversidad de experiencias migratorias y ejercicios de maternidades y paternidades, la autora afirma que "el análisis comparado se impone" (Rosas, 2013, p. 143).

\section{Trayectorias de mujeres bolivianas en el campo y en la ciudad de Mendoza}

Los relatos de vida que abordamos a continuación provienen de mujeres que tienen en común un país de nacimiento como la movilidad territorial hasta Mendoza. Asimismo, todas las entrevistadas provienen de sectores campesinos empobrecidos y comparten una imagen estereotipada asociada con una posible pertenencia étnico-aborigen (Pizarro, 2009). Debido a estos y otros motivos, han experimentado formas históricas de desigualdad en el marco de las estructuras clasificatorias de la otredad (Pizarro, 2013), tanto en Bolivia como en Mendoza, donde residen actualmente. Otra de las características 
que comparten es que se articularon en algunos segmentos precarizados del mercado de trabajo destinados a migrantes recientes (Herrera Lima, 2005), como la agricultura, el trabajo de cuidados y el comercio informal. Una de las particularidades que reviste el caso de Mendoza es que las cosechas agrícolas se presentan como la puerta de entrada al mercado de trabajo para quienes son migrantes procedentes de Bolivia y el noroeste de Argentina. En estos contextos, "los bolivianos" son estereotipados como los "más adecuados" por considerarlos "más trabajadores". En el plano simbólico, esta operatoria contribuye a justificar y naturalizar condiciones de trabajo sumamente desventajosas para las/os trabajadores (Moreno, 2013, 2015), mientras le permite a los dueños de los medios de producción abastecerse de una mano de obra barata, que asume total o parcialmente los costos de su reproducción y retiro (Meillassoux, 1985; Kearney, 2008).

Carta 1: Localización de Ugarteche (Luján de Cuyo) y Belgrano (Guaymallén)

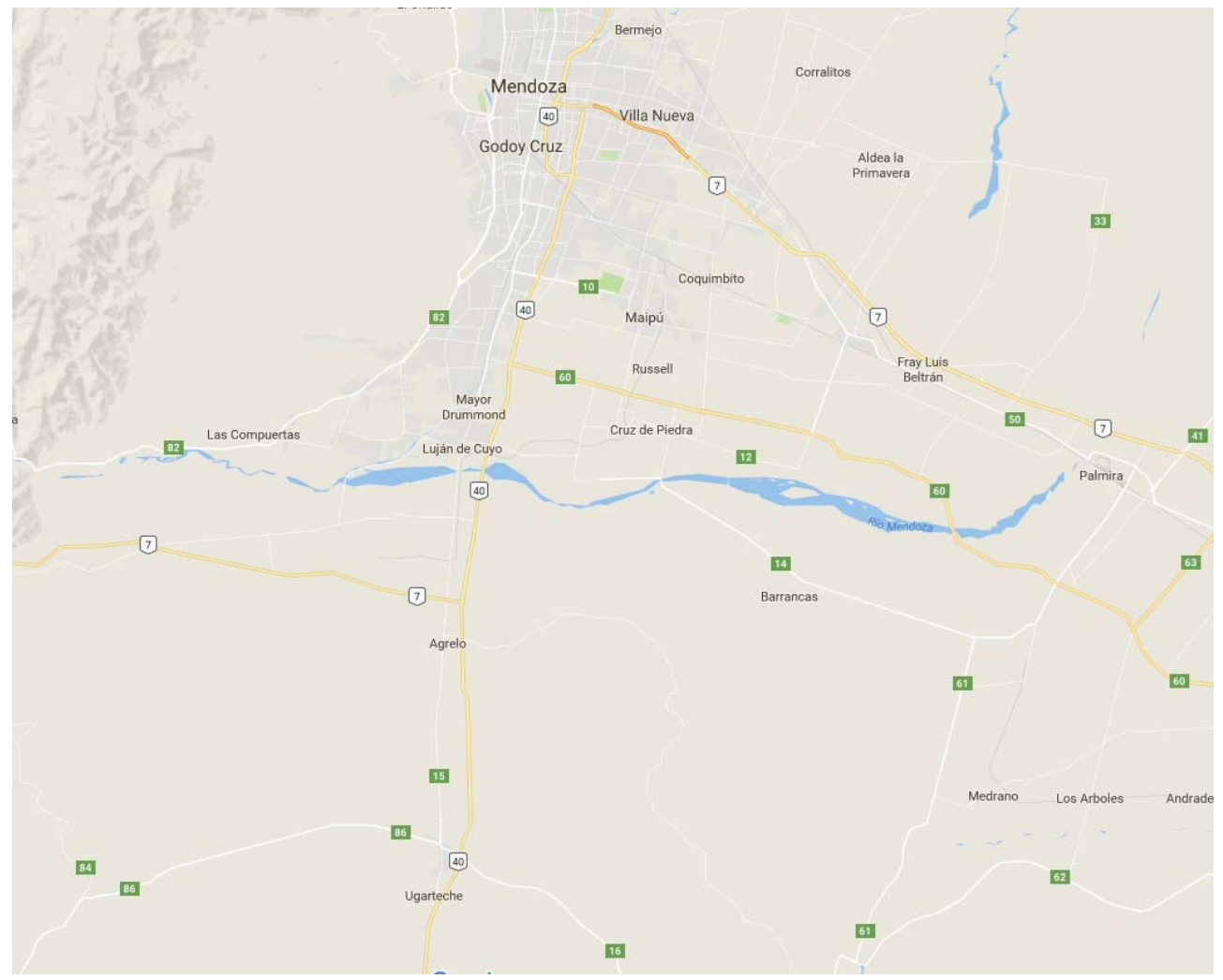

Fuente: Google Maps. 
En este marco, cabe destacar que las trayectorias de todas nuestras entrevistadas tienen en común la llegada a Mendoza para desempeñarse en la agricultura, principalmente en las cosechas estacionales de hortalizas, frutales y vid; y en menor medida, en la mediería hortícola ${ }^{5}$. Se trata en todos los casos de trayectorias marcadas por una socialización laboral temprana que ha supuesto la vivencia del trabajo como una "necesidad" desde la infancia (Mingo y Berger, 2009). Esta iniciación en el mundo del trabajo ha tenido lugar en los territorios de origen, la mayor parte de las veces a través de la colaboración o ayuda en el marco de economías familiares campesinas (Ormachea Saavedra, 2015), caracterizadas por la producción agrícola-ganadera en pequeñas propiedades, cuyos productos se destinan tanto al autoconsumo como a la comercialización por parte de los mismos productores, es decir, por ellas y los miembros de sus familias; y en menor medida a través del servicio doméstico cama adentro (como "criadas") y la venta ambulante de distintos productos de consumo (alimentos, vestimenta, cosméticos). "Yo de chiquita me pusieron trabajito, yo no conozco mi padre, así que yo vendía pan, caramelos, fruta, de todo me mandaba mi mamá" (migrante boliviana, 53 años, residente en Belgrano).

Asimismo, la mayor parte de los casos reseñados también ostenta una prolongada trayectoria migratoria, con desplazamientos por etapas o de tipo escalonado, a través de movimientos previos desde sus pueblos en Bolivia a las ciudades, y de estancias variables en otras localizaciones de Argentina, principalmente en el noroeste (Jujuy y Salta) o el centro (Córdoba, Rosario, Buenos Aires) del país, antes de arribar por primera vez a Mendoza. Se trata de una enorme variabilidad de proyectos migratorios que solo en ciertos casos revisten la forma de migraciones de grupos familiares de cónyuges con hijos. Otros resultan modalidades de reunificación familiar, que buscan el reencuentro de estas mujeres con familiares establecidos previamente en Mendoza (madre, hermana/o, tía/o, prima/o). Los desplazamientos a veces toman la forma de una circulación asociacional (Ariza, 2007; Pizarro, 2015) propiciada por varones "que las traen" para trabajar en la agricultura. "En Santa Cruz [de la Sierra/Bolivia] he conocido un paisano que me ofreció trabajo en Rosario [Argentina], en la quinta [...] en 2008 me ha traído" (migrante boliviana, 52 años, residente en Ugarteche).

5 De acuerdo con Benencia y Quaranta (2006), la mediería o "medianería" constituye una modalidad de contratación de la mano de obra extrapredial, en la que el patrón aporta la tierra, la tecnología y el capital operativo, mientras que el medianero se hace cargo de proveer la totalidad del trabajo. En estos casos, los medianeros o "chacareros a porcentaje" no reciben un salario, sino un porcentaje de las ventas de la producción a partir de las negociaciones y acuerdos a los que arriba informalmente con el patrón, por lo que constituye una modalidad de trabajo sumamente precarizada, propia de los primeros peldaños de la escalera hortícola boliviana. 
También encontramos modalidades autónomas de migración de mujeres solas o acompañadas de sus hijos o hermanas/os, que dan inicio a su proyecto migratorio luego de sufrir algunos constreñimientos generacionales o de género en el seno de sus familias. En el primer caso se trata de trayectorias migratorias que toman cuerpo, entre otras cuestiones, debido a relaciones familiares conflictivas con los progenitores. "No me llevaba con mi madre; por eso me fui jovencita" (migrante boliviana, 26 años, residente en Ugarteche). En el segundo, nos encontramos frente a mujeres que han sufrido enormes asimetrías en las relaciones de género intra-familiares, manifestándose en el abandono del marido, o bien en la opresión y explotación ejercida por el cónyuge. "Cuando es solo es solo, no pasa nada, tranquilos estamos [...] en cambio, cuando empieza 'que vos no haces nada,' 'que vos no haces', ¡eso es malísimo! Cuando tienes marido, nena, eso es todos los días" (migrante boliviana, 47 años, residente en Ugarteche).

En este sentido, podemos destacar que a pesar de que la posibilidad de "conseguir trabajo" se reitera en todas las trayectorias familiares analizadas, el abordaje de las migraciones "laborales" no debe reducirse a una evaluación única de factores económicos que se complementen con "análisis micro-estructurales basados en supuestos de individualismo extremo" (Herrera Lima, 2005, p.55). En esta línea, recuperamos los aportes de Mederios de Palma y Moraes Silva (2012), quienes sostienen que entre las determinaciones de una trayectoria más o menos compleja no pueden contabilizarse apenas los sentimientos de rebeldía contra las imposiciones económicas. "Relaciones interpersonales tensas, marcadas por cuestiones de género, raza-etnia, generación, autoridad (y, por lo tanto, poder) causan también desplazamientos" (2012, p. 74) para estas autoras.

Así, en varios de los casos analizados notamos que las relaciones generacionales y de género condicionan notablemente la decisión de emprender un proyecto migratorio. Estos resultados pueden relacionarse con la reflexión de Nadia Rizzo (2007), quien, de acuerdo con los resultados de un estudio sobre experiencias de mujeres latinas en Alemania, afirma que a través de la migración algunas mujeres pueden renegociar sus roles de género al interior de la familia y la comunidad de origen ${ }^{6}$. Por lo tanto, la migración puede constituir un movimiento intencionado para modificar patrones de género y generación desfavorables (Insa y Martínez Espínola, 2015).

6 Si bien esta perspectiva está en relación con nuestra información obtenida en campo, no pretendemos generalizar estos resultados. Asimismo, reconocemos críticamente que subyace a algunos trabajos sobre migraciones una concepción etnocéntrica según la cual las sociedades de acogida ofrecen a las mujeres migrantes grandes oportunidades para su liberación personal y su empoderamiento. Este preconcepto aparece ligado a un enfoque evolucionista basado en la idea de que los cambios en la vida de las personas migrantes se producen en las sociedades de destino, mientras que las sociedades de origen se representan carentes de historia y de una dinámica propia (Moujoud, 2008, p. 37). 
De este modo, existen numerosos factores que inciden en la construcción de los proyectos migratorios. Estos se van gestando progresivamente como una alternativa posible frente a diversos tipos de condicionamientos (de clase, género, generación, raza/etnia, etc.) que se imbrican, además, en marcos interpretativos entretejidos con el habitus migratorio (Hinojosa Gordonava, 2009), con las redes y con los imaginarios sobre los lugares de origen en diálogo con las bondades de los lugares de destino, entre otras cosas (Rivero Sierra, 2013; Pizarro, 2013). "Allá en Bolivia no es como acá. Acá hay trabajo pero de sobra, acá estamos bien, pero allá no hay nada [...] es la verdad, allá no hay futuro" (migrante boliviana, 36 años, residente en Ugarteche).

Respecto de la importancia de las redes sociales en el itinerario migratorio como en el establecimiento en la sociedad de destino, acordamos con Mallimaci cuando afirma, apoyándose en otros estudios sobre inmigración boliviana hacia la Argentina, que, en general, las mujeres que migran desde el país vecino llegan a través de relaciones familiares previas (Mallimaci, 2011, p. 755). En los casos analizados se advierte la actuación de redes familiares y amicales donde las mujeres cumplen roles protagónicos: madres, hijas, hermanas, amigas y vecinas que cooperan y también se enfrentan, se "mandan a llamar" o "van en busca", "salen", "entran" y "circulan" a veces solas, o bien junto a hijos, parientes o allegados; en fin, todas muestras corporizadas de la capacidad de proyectar y apostar en la vida que en nada se asemejan a los estereotipos patriarcales que las califican como sujetas pasivas, recluidas al ámbito de la domesticidad (Pizarro y Moreno, 2015).

Por el contrario, se trata en todos los casos de mujeres que asumen las labores productivas y reproductivas de sus familiares tanto durante el itinerario migratorio como luego de establecerse en destino. En Mendoza y en las provincias del noroeste argentino, las entrevistadas han trabajado en la cosecha como jornaleras "al tanto" (remuneradas por productividad). "Todo el tiempo trabajamos así [...] empieza la atada, nos vamos a la atada, después empieza el desbrote, vamos... vamos con el cuadrillero de acá [...] sí, trabajamos en negro" (migrante boliviana, 35 años, residente en Ugarteche). De acuerdo a los testimonios recogidos, el registro subjetivo que permanece acerca del trabajo agrícola se refiere al sacrificio que implica desde el punto de vista físico, el cual está dado por la extensión de la jornada laboral, el esfuerzo de estar agachadas bajo el sol y el hecho de no comer ni hidratarse adecuadamente.

Entre las trabajadoras que actualmente se desempeñan en la actividad, estas circunstancias son sobrellevadas a partir de argumentaciones que destacan la habituación que se obtiene con los años de práctica. "[¿¿Y usted cosecha todas las temporadas?] Sí, sí, yo cosecho todos los años. [¿ंHay que tener fuerza no?] Síii, uno se acostumbra" (migrante boliviana, 
50 años, residente en Ugarteche). Pese a que algunas trabajadoras consideran que con el tiempo "se acostumbran", ello no implica que no padezcan dolores o molestias corporales diariamente por pasar varias horas trabajando en posiciones incómodas, que implican subir escaleras con pesadas cargas en las cosechas de frutales, curvar la espalda para escardillar o plantar hortalizas, o bien agacharse o permanecer "en cuclillas" para cosechar uva en espalderos, donde se alterna entre la "quietud e incomodidad" mientras se llena el tacho y el "trote entre las hileras" cuando se lo transporta, lo que acaba causando una considerable fatiga corporal.

Sin embargo, para algunas trabajadoras agrícolas el sacrificio que envuelve esta actividad permite como contraparte obtener mejores remuneraciones en comparación con otras posibles inserciones destinadas a las mujeres, como el servicio doméstico. En una conversación capturada durante el trabajo de campo en la que participaban dos mujeres residentes en Ugarteche, una de ellas trabajadora agrícola procedente de Bolivia y la otra hija de bolivianos que trabaja en el servicio doméstico, se ponen de relieve estas consideraciones:

S: Hola, R[...] ¿En qué estás trabajando? ¿Estás cosechando?

R: No, yo estoy trabajando en una casa en Chacras; trabajo 8 horas y me vuelvo.

S: ¿Y cuánto están pagando?

R: \$1700 están pagando el mes; es poco, pero van subiendo si te quedás.

S: Uhh, es poco.

R: Lo que pasa que no me puedo el tacho.

S: Nooo, yo prefiero mil veces la viña, porque gano más, saco el doble [...] en una semana, si quiero, saco eso... igual te lo tenés que ganar, jeh! ¡Corres y corres y corres! (intercambio entre migrante boliviana de 35 años e hija de migrantes bolivianos de 25 años, ambas residentes de Ugarteche).

Vemos, así, que el servicio doméstico es una actividad en general desestimada por muchas trabajadoras agrícolas migrantes, permaneciendo como una alternativa solo en los momentos en que no surgen otras posibilidades de trabajo. En cambio, la venta ambulante o en las ferias, que tiene lugar los días domingo, resulta una opción alentadora para algunas trabajadoras agrícolas bolivianas que ya son mayores y llevan muchos años de residencia en Ugarteche. En estos casos, la exigencia física impide continuar con el desempeño en la agricultura, al tiempo que el comercio va posicionándose como una salida que tiene a las mujeres como actoras privilegiadas. 
Respecto de las migrantes que residen en zonas urbanas, vemos que la mayoría de las entrevistadas ha trabajado en la agricultura en el momento de su llegada, para desempeñarse luego en el servicio doméstico o de cuidados y en el comercio. Para las migrantes residentes en el distrito Belgrano, por lo general el servicio doméstico constituyó una experiencia breve o realizada de manera estratégica como puente para ahorrar y poder emprender luego un proyecto propio. Esto se explica porque el hecho de trabajar como vendedoras, ya sea en puestos semifijos en el centro de la ciudad o dentro de ferias comerciales, representa un claro avance en sus situaciones laborales, ya que constituye una actividad autónoma que puede realizarse con gusto, a diferencia del mayor sacrificio implicado en las experiencias laborales previas en la agricultura. Al respecto, nos parece importante señalar que la venta ambulante es una actividad que muchas de las entrevistadas conocían y practicaban ya en sus lugares de origen.

[¿Hace cuánto que usted se dedica a vender?] ¿Yo? Hace mucho... desde mis 8 años. Vendí antes en Bolivia. Fui a Santa Cruz e igual vendí. Fui a Potosí e igual vendí. Y aquí, desde el día en que llegué vendí (migrante boliviana, 49 años, residente en Belgrano).

En estos casos también se destacan algunas ventajas que ofrece este tipo de trabajo en comparación con otras posibles inserciones en el servicio doméstico o en la agroindustria. En particular se valora el mayor margen de independencia horaria propia de un trabajo "sin patrones", que además brinda la posibilidad de compatibilizar las actividades productivas con aquellas reproductivas, vinculadas con la crianza de las/os hijas/os pequeños.

[¿Y qué la llevó a realizar ese oficio, esa actividad? ¿Por qué empezó a trabajar en la venta?] Por mandar a los chicos a la escuela, por estar en la casa. Porque si voy a la fábrica, ¿quién va a mandar a los chicos a la escuela? (migrante boliviana, 61 años, residente en Belgrano).

Esta mayor autonomía que en cierta medida proporciona la venta ambulante resulta sumamente valorada por las entrevistadas. Pero, además, muchas de ellas manifiestan un gusto especial en la realización de este tipo de trabajo, dada la posibilidad de socializar con vecinos y compatriotas, "hacerse conocer en la zona" y "sentirse parte" de una comunidad.

"Ahhh, me encanta, me gusta vender verduras, tranquilo me gusta vender (migrante boliviana, 61 años, residente en Belgrano). 
Para mí la feria es parte de tu vida, parte de lo que tú haces, es la misma rutina que tienes, vas donde ya te conocen, donde ya te sientes parte de esas personas (migrante boliviana, 33 años, residente en Belgrano).

Algunas investigaciones realizadas en Bolivia por Silvia Rivera Cusicanqui y María Eugenia Choque rescatan el papel hegemónico de las mujeres en los mercados paceños no solo por su visibilidad, sino por la importancia de su rol "en la actividad productiva, organizativa y femenina a la hora de garantizar el establecimiento, supervivencia o expansión de este tipo de negocios" (Rivera Cusicanqui y Choque, 1994, en Informe Proyecto PIEB, 2008, pp. 16-17). En el contexto nacional, Benencia y Karasik (1995) analizan las ferias y comercios donde cobran protagonismos las mujeres procedentes de Bolivia. Para dichos autores, la mujer campesina boliviana cuenta, en general, con una importante experiencia en la venta, que se asocia con el buen desempeño posterior en este campo. Esta experiencia previa remite también a la aceptación social de la participación de la mujer en estas actividades y su relativa autonomía en el manejo de los recursos.

En sintonía con los aportes de estos autores, buscamos dar cuenta de algunas explicaciones que integren dimensiones históricas y culturales en la comprensión de estas actividades. De acuerdo a los relatos de algunas entrevistadas, las ferias (o "mercados del campo" en Bolivia) se desarrollaban en fechas festivas o los días domingos en "cruces" o lugares estratégicos, accesibles para varios pueblos o ayllus ${ }^{7}$. Allí los pobladores acudían para trocar o vender sus cosechas (papa, maíz, cebada), comidas típicas (comidas, bebidas) y artesanías (tejidos, cestería, instrumentos). Estos relatos muestran que la actividad de "hacer feria" es una práctica cotidiana con profundidad histórica que antecede y por tanto acompaña los procesos migratorios internos e internacionales de las mujeres procedentes de Bolivia (Colque et al., 2015; Martínez Espínola et al., 2015).

Por último, cabe mencionar que en ambos grupos las tareas no remuneradas al interior de los hogares recaen casi enteramente sobre estas trabajadoras u otras mujeres de la familia. "En la tarde llego y en la mañana me cocino un poquito y como está mi mamá; me lo cocina pa' los niños” (migrante boliviana, 35 años, residente en Ugarteche). Entre las principales actividades se destaca la preparación de alimentos, el lavado de ropa, la limpieza del hogar y el cuidado de los hijos menores, con una atención especial a su educación. "La venta para mí lo es todo para mantener a mi familia; me levanto a las 6 de

7 Ayllu (del idioma qechua): unidad política, económica y social compuesta por familias unidas por vínculos sanguíneos, ancestrales, territoriales, laborales, religiosos y de reciprocidad, existente desde tiempos anteriores al Incario (Colque et al., 2015). 
la mañana, llevo a los niños a la escuela y salgo a vender" (migrante boliviana, 27 años, residente en Belgrano).

\section{Conclusión}

En este trabajo buscamos explorar algunas de las potencialidades del análisis comparativo para profundizar la comprensión sobre las trayectorias laborales de mujeres bolivianas que residen actualmente en Mendoza y trabajan, algunas, en la agricultura en zonas rurales, y otras en la venta ambulante o en ferias emplazadas en espacios urbanos.

Hemos puesto de relieve que sus proyectos migratorios, al igual que las formas de circular y trabajar en las distintas localizaciones a lo largo de sus trayectorias, constituyen parte de proyectos migratorios familiares donde las familias migrantes, por lo general, distan de asemejarse al modelo de familia moderna corresidencial, nuclear y conyugal (Trpin y Radonich, 2013), y donde las mujeres cumplen roles protagónicos en las redes familiares $\mathrm{y}$ amicales que se construyen entre Bolivia y Argentina.

Asimismo, hemos observado que, además de la posibilidad de conseguir trabajo, existen múltiples factores que contribuyen a modelar las decisiones migratorias. En los casos de estudio seleccionados (y por cuestiones de relevancia analítica) focalizamos en algunos condicionamientos generacionales y de género que acabaron por precipitar las decisiones migratorias. Aunque también reseñamos otros aspectos, como el habitus migratorio y los imaginarios de "aquí y allá" que circulaban mediante estas redes, como elementos relevantes en la explicación de la renovación de los flujos. Tal como plantean Magliano y Mallimaci (2015), la prolongada tradición migratoria boliviana en Argentina, siempre presente como estrategia familiar, ha permitido la constitución de los movimientos como una estructura permanente entre ambos países, en que cada nueva generación de migrantes que llega al país encuentra ya establecida una comunidad de emigrantes más antiguos a la cual puede agregarse (Mallimaci, 2012, p. 181). En este marco, coincidimos con estas autoras en cuanto a que los desplazamientos migratorios no son vividos como un "quiebre" o un momento excepcional en las trayectorias vitales, sino más bien como desplazamientos de diferente grado de importancia que se superponen a lo largo del trayecto (Magliano y Mallimaci, 2015). 
Desde esta perspectiva buscamos profundizar en el mundo de los trabajos disponibles para las mujeres procedentes de Bolivia en Mendoza, deteniéndonos primeramente en las cosechas agrícolas como puerta de entrada para estas migrantes, definidas por el capital como mano de obra barata dispuesta a aceptar las condiciones de remuneración y trabajo. En este marco, encontramos que para todas ellas la actividad agrícola era representada como una labor muy exigente y sacrificada en términos psico-físicos. No obstante, el ejercicio comparativo nos permitió advertir que, entre las mujeres bolivianas que actualmente se desempeñan en este sector, el sacrificio resultaba tolerado en base a argumentaciones que destacaban la habituación que proporcionaban los años de práctica, además de los estímulos que proporcionaban los ingresos relativamente mayores que se obtenían en los períodos de cosechas, en comparación con los percibidos en otras actividades. Asimismo, se halló que el servicio doméstico resultaba una alternativa crecientemente desestimada por las trabajadoras rurales, mientras que la venta al menudeo en ferias se posicionaba como la ocupación del período de vejez entre aquellas que se habían establecido desde hacía mucho tiempo en Ugarteche.

Por su parte, entre las mujeres que en la actualidad residen en zonas urbanas y se desempeñan en la venta ambulante o en ferias dominicales advertimos el desenvolvimiento de trayectorias laborales diferentes, donde el trabajo agrícola se posicionaba solo como una actividad temporaria que efectuaron luego de su arribo, rápidamente remplazada por otras inserciones posibles, menos exigentes y sacrificadas, como el servicio doméstico. En estos casos vimos que la actividad de cuidados tampoco satisfacía las expectativas de las entrevistadas, aunque esta vez se volvía un recurso valioso para ahorrar y emprender un negocio propio en la ciudad. A diferencia de los otros trabajos destinados a migrantes recientes, encontramos que la venta constituye una actividad muy valorada por las entrevistadas, que representa un claro ascenso en sus trayectorias laborales, permitiéndoles incrementar su autonomía por el mayor margen de independencia horaria junto a la posibilidad de compatibilizar las tareas productivas y reproductivas. En estos casos también se advirtió una mayor experiencia previa en el comercio, reforzada por la imagen que representa a las mujeres andinas como "buenas vendedoras". Así, pudimos advertir que las entrevistadas reconstruyeron en los espacios urbanos los viejos paisajes de intercambio que ya eran frecuentes en sus territorios de origen. A partir de estos testimonios comprendimos que las ferias en la región andina resultan ser una institución para sus pobladores desde periodos muy antiguos.

En síntesis, el abordaje comparativo nos permitió complejizar el mapa de desplazamientos migratorios e inserciones laborales de las trabajadoras bolivianas en una provincia con una significativa antigüedad en la recepción de personas provenientes de dicho país. 


\section{Referencias}

Ariza, M. (2007). Itinerario de los estudios de género y migración en México. En: Ariza, M. y Portes, A. (coords.), El país transnacional: Migración mexicana y cambio social a través de la frontera. México: UNAM.

Ariza, M. y Oliveira, O. (2001). Familias en Transición y Marcos Conceptuales en Redefinición. Papeles de Población, número 28, Universidad Autónoma del Estado de México Toluca, pp. 9-39.

Balán, J. (1985). Migraciones internacionales en el Cono Sur (CIM). Washington: Universidad de Georgia.

Benencia, R. (2004). Apéndice: La inmigración limítrofe. En: Devoto, Fernando, Historia de la inmigración en la Argentina. Segunda Edición. Buenos Aires: Sudamericana, pp.433484.

- - - . (2008). Migrantes bolivianos en la periferia de ciudades argentinas: Procesos y mecanismos tendientes a la conformación de territorios productivos y mercados de trabajo. En: Novick, Susana (comp.), Las migraciones en América Latina. Políticas, culturas y estrategias. Buenos Aires: Catálogos, pp. 13-30.

Benencia, R. y Karasik, G. (1995). Inmigración limítrofe: los bolivianos en Buenos Aires. Buenos Aires: CEAL.

Benencia, R. y Quaranta, G. (2006). Mercados de trabajo y relaciones sociales: la conformación de trabajadores agrícolas vulnerables. Sociología de Trabajo (nueva época) $\mathrm{N}^{\circ} 58$. Madrid: Siglo XXI.

Candau, J. (2002). Antropología de la memoria. Buenos Aires: Nueva Visión.

Cavagnaro, J. y Balussi, M. A. (1962). Estudio sociológico sobre los grupos migratorios de braceros bolivianos en Mendoza. Cuadernos de Instituto de Estudios Políticos y Sociales 8.

Colque, N., Martínez Espínola, M. V., Moreno, M. S. y Sánchez, M. (2015). “Representaciones en tensión: Análisis de registros fotográficos de las movilizaciones realizadas por los feriantes de Guaymallén (Mza, Arg.) durante el año 2014". Ponencia presentada en las XI Jornadas de Sociología de la UBA Cuerpos, tiempos, saberes. 
Comas D’Agemir, D. (1998). Trabajo; género y cultura. La construcción de la desigualdad entre hombres y mujeres. Barcelona: Icaria-Institut Catalá d’ Antropologia.

García Vazquez, C. (2005). Los migrantes. Otros entre nosotros. Etnografía de la población boliviana en la provincia de Mendoza. Mendoza: EDIUNC.

Garza Toledo, E. (2002). Problemas clásicos y actuales de la crisis del trabajo. En Enrique de la Garza Toledo y Neffa (comp.), El futuro del trabajo, el trabajo del futuro. Buenos Aires: CLACSO.

Gregorio Gil, C. (2011). "Análisis de las migraciones transnacionales en el contexto español, revisitando la categoría de género desde una perspectiva etnográfica y feminista". Trabajo final del proyecto SEJ2005-0639: Desigualdades de género en el contexto de la globalización: cuidados, afectos y sexualidad, financiado por el Plan Nacional de Investigación, Desarrollo e Innovación Tecnológica de la Secretaría de Estado de Universidades e Investigación. Universidad de Granada, España.

Hammersley, M. y Atkinson, P. (1994). Etnografía. Métodos de investigación. Buenos Aires: Paidós.

Harris, O. (1986). La unidad doméstica como unidad natural. Nueva Antropología, vol. VII, núm. 30, pp. 199-222.

Herrera Lima, F. (2005). Vidas itinerantes en un espacio laboral transnacional. México: Universidad Autónoma Metropolitana.

Hinojosa Gordonava, A. (2009). Buscando la vida. Familias bolivianas transnacionales en España. La Paz: CLACSO-Fundación PIEB.

Hondagneu-Sotelo, P., Estrada, E. y Ramírez, H. (2011). Más allá de la domesticidad. Un análisis de género de los trabajos de los inmigrantes en el sector informal. Papers 96(3), pp. 805-824.

Insa, C. y Martínez Espínola, V. (2015). Trayectorias laborales de mujeres peruanas y bolivianas en el Área Metropolitana de Mendoza. Iberoamérica Social. Revista - red de estudios sociales, año III, No4, pp. 55-72. 
Kearney, M. (2008). Lo global y lo local: La antropología de la globalización y el transnacionalismo. En: Hiernaux, D. y Zárate, M. (eds.). Espacios y transnacionalismo. México: UAM-JP.

Kornblit, A. L. (2007). Historias y relatos de vida: una herramienta clave en metodologías cualitativas. En: Kornblit, A. L. (comp.), Metodologías cualitativas en ciencias sociales. Buenos Aires: Biblos, pp.15-34.

Magliano, M. J., Perissinotti, M. V. y Zenklusen, D. (2013). Mujeres bolivianas y peruanas en la migración hacia Argentina: especifcidades de las trayectorias laborales en el servicio doméstico remunerado en Córdoba. En Anuario Americanista Europeo 11, pp.71-91.

Magliano, M. J. y Mallimaci, A. (2015). Las edades de la migración boliviana en Argentina: Córdoba y Ushuaia como destino. Si Somos Americanos. Revista de Estudios Transfronterizos, volumen XV, $\mathrm{N}^{\circ} 1$, pp. 141-167.

Mallimaci, A. (2011). Migraciones y géneros. Formas de narrar los movimientos por parte de migrantes bolivianos/as en Argentina. Revista Estudos Feministas 19(3): 751-775.

- - — - (2013). Localizando el sentido de las desigualdades. Inclusiones y exclusiones de los/as bolivianos/as en Ushuaia. En: Karasik, G. (coord.), Migraciones internacionales: reflexiones y estudios sobre la movilidad territorial contemporánea. Buenos Aires: CICCUS, pp. 87-107.

Marcus, G. (2001). Etnografía en/del sistema mundo. El surgimiento de la etnografía multilocal. Alteridades 11(22), pp. 111-127.

Martínez Espínola, M. V. (2010). Experiencias migratorias de mujeres bolivianas residentes en Mendoza (Tesina de grado). Mendoza, Universidad Nacional de Cuyo. Facultad de Ciencias Políticas y Sociales. Dirección URL del informe: http://bdigital.uncu.edu. $\operatorname{ar} / 4469$.

Martínez Espínola, M. V., Moreno, M. S., Colque, N. y Sánchez, M. (2015). “Ferias, trabajo y migración: una exploración conceptual a partir del caso de las/os feriantes del distrito de Belgrano, Guaymallén, Mendoza”. Ponencia presentada en las Segundas Jornadas de Sociología de la FCPyS de la UNCuyo. «Balances y desafíos de una década larga (2001-2015): aportes y debates desde la Sociología». Realizadas en la Universidad Nacional de Cuyo, Facultad de Ciencias Políticas y Sociales, 27 y 28 de agosto. 
Martínez Espínola, Victoria (2016). Género e identidades. Una aproximación a partir de representaciones de mujeres bolivianas residentes en Mendoza. En prensa, en: VVAA, Identidad(es) socio-cultural(es) en la filosofía y el arte. Desde los espacios locales a los escenarios latinoamericanos (en prensa). Mendoza: EDIUNC.

Medeiros de Melo, B. y Moraes Silva, M. A. (2012). Trayectorias migratorias: trabajadores rurales entre el nordeste y los cañaverales de Sao Pablo/Brasil. En Roberto Benencia, Fernando Herrera Lima y Elaine Levine (coords.), Ser migrante latinoamericano, ser vulnerable, trabajar precariamente. Barcelona: Anthropos Editorial; México: Universidad Autónoma Metropolitana.

Meillassoux, C. (1985). Mujeres, graneros y capitales. Economía doméstica y capitalismo. México: Siglo XXI.

Mingo, E. y Berger, M. (2009). Asalariados rurales en el Valle de Uco (Mendoza, Argentina). Mundo Agrario vol. 10, N 19, La Plata, CEHR, Universidad Nacional de La Plata.

Moraes Silva, M.A. y Menezes, M. (2011). Migrantes temporales: resignificación de las narrativas. En Historia, Voces y Memoria 3, pp. 19-48.

Moujoud, N. (2008). Effets de la migration sur les femmes et sur les rapports sociaux de sexe. Au-delà des visions binaires. En Falquet, Jules, Rabaud, Aude et al., Femmes, genre, migrations et mondialisation: un état de problématiques, Les cahiers du CEDREF, N¹6, París: Université Paris Diderot, pp. 36-49.

Moreno, M.S. (2012). Relaciones intraétnicas en el mercado de trabajo rural a parir de una coyuntura histórica en Mendoza. Kula. Antropólogos del Atlántico Sur, vol. 2, N7, pp. 67-80.

_-_—. (2013). "Humildes, sumisos y trabajadores", ponencia presentada en XRAM, Reunión de Antropólogos del MERCOSUR, Córdoba.

- _- - (2015). Territorios migratorios: reflexiones a partir de un estudio de caso multilocal en las cosechas agrícolas de Mendoza. Ponencia presentada en las Segundas Jornadas de Sociología de la FCPyS de la UNCuyo. «Balances y desafíos de una década larga (2001-2015): aportes y debates desde la Sociología». Realizadas en la Universidad Nacional de Cuyo, Facultad de Ciencias Políticas y Sociales, 27 y 28 de agosto. 
Moreno, M.S. y Torres, L. (2013). Movimientos territoriales y dinámicas laborales: los migrantes bolivianos en la agricultura de Mendoza (Argentina). CRITERIOS. Cuadernos de Ciencias Jurídicas y Política Internacional, vol. 6, №1.

Narotzky, S. (2004). Antropología económica. Nuevas tendencias. Barcelona: Melusina.

Núñez Borja, C. y Stallaert, C. (2013) Mujeres migrantes andinas en Bruselas: género y colonialidad del poder. En Anuario Americanista Europeo 11, pp. 31-50.

Ormachea Saavedra, E. (2015). Pequeña y gran producción agrícola capitalista y trabajo asalariado en Bolivia. En Alberto Riella y Paola Mascheroni (comp.), Asalariados Rurales en América Latina. Consejo Latinoamericano de Ciencias Sociales (CLACSO), Departamento de Sociología-FCS-UDELAR.

Padilla, B. (2013) Género y Migraciones: Nuevas reconfiguraciones y protagonismos de las mujeres latinoamericanas. A modo de introducción. Anuario Americanista Europeo 11 , pp. 1-9.

Paredes, A. (2004). Los inmigrantes en Mendoza. En Rosignoli, A.I. et al., Mendoza, Cultura y Economía. Buenos Aires: Caviar Blue.

Parella, S. (2007). Los vínculos afectivos y de cuidado en las familias transnacionales: Migrantes ecuatorianos y peruanos en España. Migraciones Internacionales, vol. 4, No2, pp. 151-188.

Pedreño Cánovas, A. (2006). ¿Cómo se lo monta la otra mitad? Economía informal y estrategias de trabajo en los relatos de vida de la inmigración extranjera en España. Estudios Migratorios Latinoamericanos, año 20, №60.

Piore, M. (1979). Birds of Passage: Migrant Labor in Industrial Societies. Cambridge: Cambridge University Press.

Pizarro, C. (2006). Ahora ya somos civilizados. La invisibilidad de la identidad indígena en un área rural del Valle de Catamarca. Córdoba: EDUCC-Universidad Católica de Córdoba. Colección Thesys, vol. 10.

_-_—. (2009), “Olor a negro”. Discurso, discriminación y segmentación étnica en el lugar de trabajo. Ponencia presentada en IV Coloquio de la Asociación Latinoamericana de Análisis del Discurso, Escuela de Lengua UNC: Córdoba. 
- - - . (ed.) (2011). Ser Boliviano en la Región Metropolitana de la ciudad de Córdoba. Localización socio-espacial, mercado de trabajo y relaciones interculturales. Universidad Católica de Córdoba.

- - - . (2013). Partir y volver, yendo de Bolivia a Argentina. Ponencia presentada en las VII Jornadas Santiago Wallace de Investigación en Antropología Social, del 27 al 29 de noviembre. Facultad de Filosofía y Letras, UBA, Buenos Aires, Argentina.

- (2015). Interseccion of Inequalities: Migratory Trajectories, Labor Experiences, and Family Life of Bolivian Women on the Outskirts of Buenos Aires and Córdoba. En Cynthia Pizarro (ed.), Bolivian Labor Immigrants' Experiences in Argentina. Estados Unidos: Lexington Books.

Pizarro, C. y Trpin, V. (2010). Trabajadores frutícolas y hortícolas en la Argentina. Una aproximación socio-antropológica a las prácticas de reproducción y de resistencia de las condiciones laborales. $R U R I S$, vol. $4, \mathrm{~N}^{\circ} 2$.

Pizarro, C. y Moreno, M. S. (2015), Differential Migration Pathways of Bolivian Women Working in Horticultural Fields in Mendoza. En Cynthia Pizarro (ed.), Bolivian Labor Immigrants' Experiences in Argentina. Estados Unidos: Lexington Books.

Rosas, C. (2013) Discusiones, voces y silencios en torno a las migraciones de mujeres y varones latinoamericanos. En Anuario Americanista Europeo 11, pp.127-148.

Rivera Cusicanqui, S. (2010). Violencias (re)encubiertas en Bolivia. La Paz: Editorial piedra rota, p. 236.

Rivera Cusicanqui, S. y Choque, M.E. (1994). PROGRAMA DE INVESTIGACIÓN ESTRATÉGICA EN BOLIVIA (2008). Espacio compartido: espacio urbano y comercio informal en la ciudad de La Paz. Informe de medio término. En <http://www.pieb.org/ espacios/archivos/avance_del_proyecto_texto.pdf>.

Rivero Sierra, F. (2013). Mi papá se fue pero va a volver. Experiencias de la migración entre los no migrantes. El caso del Barrio Plan 3000 en Santa Cruz de la Sierra, Bolivia. Ponencia presentada en XRAM Reunión de Antropólogos del MERCOSUR, Córdoba.

Rizzo, N. (2007). Género y migración: sentidos e impactos de la experiencia migratoria en las biografías de mujeres latinas en Alemania. Forum Qualitative Sozialforschung /Forum: Qualitative Social Research, vol. 8, N³, art. 13. 
Sassone, S. M. (2009). Breve geografía histórica de la migración boliviana en la Argentina. En: Temas de patrimonio cultural No24: Buenos Aires Boliviana. Migración, construcciones identitarias y memoria. Buenos Aires: Comisión para la Preservación del Patrimonio Histórico Cultural de la Ciudad Autónoma de Buenos Aires, pp. 389-402.

Scott, J. (1990). Historia y género: las mujeres en la Europa Moderna y Contemporánea. Edicions Alfons el Magnanim, Institució Valenciana d’Estudis i lnvestigació.

Trpin, V. y Radonich, M. (2013), Mujeres migrantes en la organización de territorios rurales en el Alto Valle de Río Negro. En Karasik, Gabriela (coord.), Migraciones internacionales, reflexiones y estudios sobre la movilidad territorial contemporánea. Buenos Aires: CICCUS.

Whiteford, S. (2001) Articulación social y poder: el zafrero y el contexto de la plantación azucarera”. En Hermitte, E. y Bartolomé, L. (comp.) Procesos de Articulación Social. Buenos Aires: Amorrortu.

\section{Otras fuentes consultadas}

Censos Nacionales de Población, Hogares y Viviendas de Argentina 1980-1991-2001-2010. Disponible en <http://www.indec.gov.ar/> 